\title{
Principal Component Analysis and Character Association for Yield Components in Rice (Oryza sativa L.) Genotypes of Salt Tolerance under Alkaline Condition
}

\author{
A. Kashyap and Vijay Kumar Yadav* \\ Department of Genetics and Plant Breeding Chandra Shekhar Azad University of Agriculture \\ \& Technology, Kanpur- (U.P.) India \\ *Corresponding author
}

Keywords

Principal

Component

Analysis,

Genotypes,

Correlation, Salt

tolerance, Rice,

Alkaline

Article Info

Accepted:

07 September 2020

Available Online:

10 October 2020

\begin{abstract}
A B S T R A C T
The present research work was conducted at high and normal salt stress soil condition to study the inherent genetic variation existing among the 78 accessions including seven standards (checks) varieties viz., CSR36, CSR10, CST7-1, CSR27, Usar Dhan 3 for salinity and alkalinity tolerant, while Sambha Sub1 as for general stress, and PUSA 44 as salt stress sensitive were grown in Augmented Randomized Block Design as parents for developing new rice varieties to break the yield barrier under sodic land. Grain yield showed highly significant and positive association ship with filled grains panicle ${ }^{-1}$ followed by spikelet fertility, plant height, and test weight can be used as selection criteria for yield improvement under high stress environment. Principal Component Analysis revealed that first three principal components addressed $80.038(\%)$ of the total variability which was represented by the first five principal components. CARI Dhan 11, CSRC(S) 47-7-B-B-1-1, IR52280-117-1-1-3, CSR 2016-IR18-10, CSA 2016-IR18-6 6 had high Principal Factor score in factor 1 indicated that they had high variation for spikelet fertility (\%). Similarly, Principal Factor score for NDRK 11-24, KR15006, NDRK 11-21, CSR36 (C), Usar Dhan 3 (C) were showed high variation in factor 2 which representing the productive tillers plant-1, 1000 seed weight.
\end{abstract}

\section{Introduction}

More than half of the world populations depend on rice for their survival. It plays a pivotal role in food security of many countries. Rice crop is interwoven in the cultural and social life of millions of Asian. Salinity and alkalinity are growing problems worldwide. It can either be natural or primary or secondary or men made salinity. Primary salinity of soil and ground water is due to the weathering of naturally saline rocks or by deposition of oceanic salt carried by the wind and rain affects $130 \mathrm{~m}$ ha out of the global land area (Szaboles, 1994). Millions of hectares land in the humid regions of south and Southeast Asia are technically suited for rice production but are left uncultivated or are grown with very low yields because of salinity and other soils problem. Salinity is a growing problem worldwide. It can either be natural or primary or secondary or men made 
salinity. Primary salinity of soil and ground water is due to the weathering of naturally saline rocks or by deposition of organic salt carried by the wind and rain. Secondary salinity is due to human-activities like unreliable irrigated schemes and source of water. Successive salinity in the soil has the divesting effect on plant growth reducing crop yields worldwide that leads complete crop failure in worst affected areas.

Various abiotic stresses i.e. flood, drought, and salinity and biotic stresses like pests and diseases are needs to more attention because they are directly hurdle in rice production. Among the abiotic stresses, drought is the strongest constraint, affecting nearly a third of the total rice area in Asia and causing significant economic losses to poor rice producers in the region. Salinity, though not as big a stress as drought and flood, still affects $10 \mathrm{~m}$ ha of coastal and inland areas. But, growing rice is the only option available to farmers in the saline-prone coastal regions where nothing else can be grown, and this crop is critical to the food security of these resource-poor farmers.

Prior to identifying new an approach to break the yield barrier, it is pertinent to study the correlation for yield and its components in the rice germplasm suitable for sodicity and clustering them into different groups based on the diversity among the accessions. Principal component analysis is a statistical procedure which commonly used for compression, reduction and transformation of data. The original large data set, which involves many variable just a few variable called the principal components. In other words, principal component analysis (PCA) transforms a number of possibly correlated variables into a smaller number of uncorrelated variables called principal component. This technique assist in identification of traits that help in distinguishing selected genotypes based on similarities in one or more traits and classify the genotypes into separate groups (Ariyo, 1987 and Nair et al., 1998). PCA is use to identify the traits with high variability; correlations reveal the strength of relationship between various yield and its components. Trait association assist the breeder to understand the mutual component traits on which selection can be based for genetic improvement (Chakravorty et al., 2013, Ravikumar et al., 2015). Information on correlation, direct and indirect effects contributed by each character towards yield will be an extra advantage in aiding the selection process and helps the breeder to design his selection strategies for improving the grain yield (Ravindra Babu et al., 2012). The present investigation is carried out "Principal Component Analysis and character association for yield components in rice (Oryza sativa L.) genotypes of salt tolerance under alkaline condition".

\section{Materials and Methods}

The experiment was conducted during kharif 2017 and 2018 as transplanted irrigated rice at Crop Research Farm, Nawabganj and Seed Multiplication Farm Bojha, Chandra Sheker Azad University of Agriculture and Technology, Kanpur (U.P.) India. The experimental materials comprising of 71 rice genotypes and seven standards (checks) varieties viz., CSR36, CSR10, CST7-1, CSR27, Usar Dhan 3 for salinity and alkalinity tolerant while, Sambha Sub1 as for general stress, and PUSA44 as salt stress sensitive were grown in Augmented Randomized Block Design with replications of check. The experiment was conducted in three environments taking into consideration of soil types and days of sowing. E-1: Environment I, Year 2017, high stress, pH 9.8, Ec $1.43 \mathrm{dsm}^{-1}$, Seed Multiplication Farm, Bojha, E-2:Environment II, Year 2018, high 
stress, $\mathrm{pH}$ 9.8, Ec1.41 $\mathrm{dsm}^{-1}, \quad$ Seed Multiplication Farm, Bojha,E-3: Environment III, Year 2018, Normal stress, pH 8.8, Ec0.96 $\mathrm{dsm}^{-1} \mathrm{CRF}$, Nawabganj. Each genotype was grown in seven-row of $01 \mathrm{~m}$ length following $15 \mathrm{~cm}$ row to row and plant to plant spacing. Recommended package of practices was adopted to raise a healthy crop. Data was recorded from ten selected plants on traits viz., days to $50 \%$ flowering, plant height (cm), Total No. of tillers plant ${ }^{-1}$,number of panicle bearing tillers plant ${ }^{-1}$,panicle length (cm),filled grain panicle ${ }^{-1}$,spikelet fertility percentage,1000 - grain weight (g),stress score at reproductive stage, grain yield per plant, and the mean data was used for statistical analysis.

\section{Results and Discussion}

Analysis of variance of all the traits except panicle length and filled grains panicle $^{-1}$ under study was found to be highly significant. Days to 50 percent flowering ranged from 77.0 (CSR-2748-4441-193) to 127.33 (CR 2851-S-B-1-B-B-1) with a mean of 99.19 days. The plant highest varied from $63.86 \mathrm{~cm}$ (TR 09027) to 153.83(CARI Dhan 11)) with mean value of $94.12 \mathrm{~cm}$. The highest total tillers plant $^{-1}$ was recorded in CSR-2748-197(17.6), while lowest in IR84649-81-4-1-3B-CR3397-S-B-4(7.23)

with mean of 12.56 . The average number of productive tillers plant ${ }^{-1}$ was 9.74 with highest 14.73 in CSR10 and lowest in IR84649-81-41-3B-CR3397-S-B-4(5.5). The panicle length ranged from $17.48 \mathrm{~cm}$ (KR 15016) to 26.03 $\mathrm{cm}$ (CSR 2016-IR18-12) with mean of 21.63. Filled grains panicle ${ }^{-1}$ was found highest in RP-320-4-3-2-1(156.33) and lowest in CSR 2016-IR18-16(64.66) with mean of 117.44. Spikelet Fertility \% was varied from 65.84(CSR 2016-IR18-16) to 87.19 (CSA 2016-IR18-6) with mean value of 77.04. The highest test weight was recorded in NDRK 11-21(27.23), while lowest in CR 2851-S-B-
1-B-B-1(17.20) and mean of 21.77 gram. Thirteen genotypes were showed highest Stress score at Reproductive Stage. These were observed as very sensitive to alkalinity. Out of 78 genotypes, only five entries were found tolerant to high salt stress. The highest grain yield per plant was recorded in $\mathrm{KS}$ 12(38.12 gram) and lowest in CR 2851-S-B1-B-B-1(8.58 gram) (Table 1 and 2).

\section{Correlation studies}

To find out the relationship existing among the characters under study with grain yield plant $^{-1}$ and its associated characters were estimated (Table 3). Genotypic correlation coefficient between most of the characters was higher in magnitude than the phenotypic correlation coefficients indicating strong association between various characters under study and the genotypic expression of the association was comparatively less influenced by the environmental fluctuations than phenotypic. The economic trait i.e. grain yield showed highly significant and positive association ship with filled grains panicle ${ }^{-1}$ followed by spikelet fertility, plant height, 1000-grain weight. Therefore, these characters emerged as most important associates of grain yield in rice under high stress condition (Nayak et al., 2001, Qamar et al., 2005; Babar et al., 2009; Jayasudha and Sharma 2010; Yadav et al., 2011; Yadav et al., 2012; Ratna et al., 2015; Akhilesh Kumar Yadav et al., 2016 and Kumar et al., 2018).

Test weight was exhibited positive association with plant height $(\mathrm{cm})$, tiller plant $^{-1}$, productive tillers plant $^{-1}$, panicle length $(\mathrm{cm})$, spikelet fertility and grain yield at both level of genotype and phenotype level which augurs well for providing correlated response during selection for improving these characters. The above observations of strong positive associations between yield and yield components are in agreement with the 
available literature in rice (Yadav et al., 2011; Yadav et al., 2012; Ratna et al., 2015; Akhilesh Kumar Yadav et al., 2016; Kalyan et al., 2017 and Prakash et al., 2018.

Significant and positive association of spikelet fertility (\%) was observed with plant height $(\mathrm{cm})$, tiller plant ${ }^{-1}$, productive tillers plant $^{-1}$, filled grains panicle ${ }^{-1}$, test weight, and grain yield at both genotypic and phenotypic level that yield can be improve simultaneously (Yadav et al., 2011; Yadav et al., 2012; Akhilesh Kumar Yadav et al., 2016; Kalyan et al., 2017; and Prakash et al., 2018). Grains per panicle showed positive and significant relationship with plant height $(\mathrm{cm})$, tiller plant $^{-1}$, panicle length $(\mathrm{cm})$, spikelet fertility, and grain yield at both genotypic and phenotypic level that these yield contributing traits can be improve simultaneously (Yadav et al., 2012; Ratna et al., 2015; Akhilesh Kumar Yadav et al., 2016; Kalyan et al., 2017; Prakash et al., 2018).

Panicle Length $(\mathrm{cm})$ reflected positive relationship with plant height $(\mathrm{cm})$ tiller plant ${ }^{-1}$, productive tillers plant ${ }^{-1}$, filled grains panicle $^{-1}$, test weight, and grain yield at both genotypic and phenotypic level that these yield contributing traits can be improve simultaneously (Yadav et al., 2011; Manikya and Reddy, 2011; Ratna et al., 2015 and Prakash et al., 2018).

Productive tillers plant ${ }^{-1}$ exhibited positive relationship with plant height $(\mathrm{cm})$, tiller plant $^{-1}$, filled grains panicle ${ }^{-1}$, panicle length, filled grains panicle ${ }^{-1}$, spikelet fertility (\%), test weight, and grain yield at both genotypic and phenotypic level that these yield contributing traits can be improve simultaneously (Yadav et al., 2012, Ratna et al., 2015; Akhilesh Kumar Yadav et al., 2016; Kalyan et al., 2017; Prakash et al., 2018). Thus, filled grains panicle ${ }^{-1}$; spikelet fertility, plant height, 1000-grain weight can be used as selection criteria for yield improvement under high stress environment.

\section{Principal component analysis}

To find out independent impact of all the traits under study principal component analysis was conducted. The first five components in the PCA analysis with Eigen values > 1 contributed 80.032 percent variability existing in the rice varieties for yield contributing characters in the study explained by the first five axes with an Eigen value of greater than unity indicates that, the identified traits within the axes exhibited great influence on the phenotype of germplasm lines (Table 4 and Figure 1). Remaining components with Eigen values $<1$ contributed 19.968 percent variability. Principal component 1 (PC 1) with Eigen value 3.349 contributed 33.485 percent of total variability. PC 2 with Eigen value of 1.375 and PC 3 with Eigen value 1.175 contributed 13.752 percent and 11.750 percent to total variability respectively. It was concluded that maximum variation was present in first PC. So selection of genotypes from this PC will be useful.

The differentiation of the genotypes into different clusters was because of relatively high contribution of few characters rather than small contribution from each character.

The positive and negative loading show the presence of positive and negative correlation trends between the components and the variables. Therefore, the above mentioned characters which load high positively or negatively contributed more to the diversity and they were the ones that most differentiated the clusters (Vishnu et al., 2014; Ravi Kumar et al., 2015 and Gour et al., 2017). 
Table.1 Mean performance of 78 genotypes for 10 characters in Oryza sativa L in three environments

\begin{tabular}{|c|c|c|c|c|c|c|c|c|c|c|c|}
\hline $\begin{array}{l}\text { S. } \\
\text { No. }\end{array}$ & Character & $\begin{array}{c}\text { Days to } \\
50 \% \\
\text { flowering }\end{array}$ & $\begin{array}{l}\text { Plant } \\
\text { height } \\
\text { (cm) }\end{array}$ & Tillers/Plant & $\begin{array}{c}\text { Productive } \\
\text { Tillers/plant }\end{array}$ & $\begin{array}{l}\text { Panicle } \\
\text { Length } \\
\text { (cm) }\end{array}$ & $\begin{array}{c}\text { Filled } \\
\text { grains/panicle }\end{array}$ & $\begin{array}{c}\text { Spikelet } \\
\text { Fertility } \\
(\%)\end{array}$ & $\begin{array}{c}\text { Test } \\
\text { Weight }\end{array}$ & $\begin{array}{c}\text { Stress score } \\
\text { at } \\
\text { reproductive } \\
\text { stage }\end{array}$ & $\begin{array}{c}\text { Grain } \\
\text { Yield } \\
\text { g/plant }\end{array}$ \\
\hline 1 & CARI Dhan 10 & 85.33 & 82.90 & 8.53 & 5.67 & 19.06 & 149.33 & 76.28 & 19.00 & 2.00 & 31.46 \\
\hline 2 & CARI Dhan 11 & 85.33 & 153.83 & 15.83 & 12.43 & 23.39 & 134.67 & 82.40 & 20.73 & 1.00 & 36.08 \\
\hline 3 & CARI Dhan 6 & 104.00 & 105.63 & 11.10 & 9.17 & 20.92 & 130.67 & 83.92 & 23.40 & 2.00 & 27.45 \\
\hline 4 & CR 2851-S-1-6-B-B-4 & 121.33 & 76.67 & 10.93 & 8.93 & 21.83 & 105.67 & 74.83 & 19.67 & 7.00 & 12.35 \\
\hline 5 & CR 2851-S-B-1-2B-1 & 96.67 & 92.07 & 15.03 & 11.27 & 19.55 & 122.67 & 75.10 & 23.47 & 3.00 & 27.00 \\
\hline 6 & CR 2851-S-B-1-B-B-1 & 127.33 & 104.53 & 14.33 & 10.67 & 21.07 & 96.00 & 71.54 & 22.68 & 7.00 & 8.58 \\
\hline 7 & CR $3437-1 *-S-200-83-1$ & 105.00 & 90.17 & 11.50 & 8.43 & 23.33 & 138.67 & 77.91 & 17.20 & 5.00 & 22.28 \\
\hline 8 & CR 3878-245-2-4-1 & 95.33 & 102.00 & 10.70 & 9.27 & 22.09 & 123.67 & 75.44 & 20.82 & 3.00 & 28.48 \\
\hline 9 & CR 3880-10-1-9-2-2-1 & 101.00 & 79.37 & 11.57 & 6.37 & 18.81 & 114.00 & 73.88 & 19.80 & 3.00 & 24.16 \\
\hline 10 & CR 3881-4-1-3-7-2-3 & 114.00 & 112.10 & 13.50 & 10.03 & 21.14 & 121.00 & 73.37 & 19.97 & 7.00 & 12.88 \\
\hline 11 & CR 3883-3-1-5-2-1-2 & 94.00 & 116.20 & 7.70 & 5.60 & 20.22 & 97.67 & 72.50 & 23.47 & 3.00 & 23.92 \\
\hline 12 & CR 3884-244-8-5-11-1-1 & 99.33 & 67.40 & 11.33 & 7.67 & 22.11 & 104.00 & 78.89 & 22.57 & 7.00 & 9.47 \\
\hline 13 & CR 3887-15-1-2-1 & 91.67 & 116.20 & 11.07 & 9.53 & 19.25 & 113.67 & 71.53 & 17.90 & 2.00 & 31.41 \\
\hline 14 & CR 3890-35-1-3-4 & 104.33 & 113.07 & 14.67 & 10.20 & 24.99 & 132.67 & 80.89 & 22.70 & 2.00 & 35.92 \\
\hline 15 & CR 3904-162-1-5-1 & 125.33 & 97.07 & 7.33 & 5.67 & 22.75 & 94.67 & 69.56 & 22.33 & 7.00 & 10.87 \\
\hline 16 & CR3881-M-3-1-5-1-1-1 & 94.33 & 98.10 & 13.33 & 10.20 & 22.52 & 115.00 & 71.22 & 20.28 & 3.00 & 30.96 \\
\hline 17 & CR3882-7-1-6-2-2-1 & 92.67 & 77.47 & 10.27 & 8.07 & 24.57 & 126.67 & 78.47 & 19.01 & 3.00 & 24.57 \\
\hline 18 & CR3884-244-8-5-6-1-1 & 100.67 & 96.68 & 15.10 & 10.27 & 22.13 & 111.67 & 77.62 & 18.82 & 7.00 & 14.58 \\
\hline 19 & CR3903-161-1-3-2 & 119.33 & 104.67 & 10.33 & 8.07 & 18.93 & 114.67 & 76.93 & 22.67 & 6.33 & 19.95 \\
\hline 20 & CSA 2016-IR18-6 & 91.00 & 113.87 & 13.17 & 11.93 & 20.87 & 149.00 & 87.20 & 22.83 & 1.00 & 36.72 \\
\hline 21 & CSAR 1604 & 92.33 & 78.23 & 10.33 & 7.60 & 19.82 & 122.67 & 73.28 & 24.60 & 3.00 & 27.81 \\
\hline 22 & CSAR 1610 & 91.33 & 90.20 & 11.77 & 9.00 & 20.43 & 112.67 & 68.92 & 23.73 & 2.00 & 29.88 \\
\hline 23 & CSAR 1628 & 105.33 & 104.51 & 9.93 & 7.90 & 19.67 & 78.67 & 67.50 & 24.03 & 5.00 & 18.94 \\
\hline 24 & CSAR1620 & 96.67 & 83.23 & 13.50 & 11.87 & 20.99 & 151.67 & 84.42 & 23.30 & 2.00 & 36.77 \\
\hline 25 & CSR 2016-IR18-1 & 96.67 & 75.53 & 12.00 & 9.33 & 21.61 & 119.67 & 76.69 & 21.54 & 7.00 & 16.41 \\
\hline 26 & CSR 2016-IR18-10 & 94.33 & 121.23 & 13.80 & 11.00 & 19.48 & 123.67 & 79.85 & 22.62 & 1.00 & 37.10 \\
\hline 27 & CSR 2016-IR18-11 & 108.33 & 103.07 & 13.37 & 10.17 & 19.75 & 120.67 & 76.64 & 20.83 & 5.00 & 18.28 \\
\hline 28 & CSR 2016-IR18-12 & 95.33 & 103.20 & 12.03 & 9.03 & 26.03 & 103.67 & 72.35 & 24.37 & 3.00 & 31.87 \\
\hline 29 & CSR 2016-IR18-14 & 114.00 & 77.28 & 12.87 & 10.93 & 19.90 & 108.33 & 77.49 & 18.97 & 6.67 & 12.34 \\
\hline 30 & CSR 2016-IR18-15 & 105.00 & 88.70 & 13.23 & 8.80 & 24.37 & 105.67 & 71.78 & 21.30 & 3.00 & 25.74 \\
\hline 31 & CSR 2016-IR18-16 & 105.67 & 84.03 & 12.43 & 7.67 & 24.97 & 64.67 & 65.84 & 22.48 & 3.00 & 19.41 \\
\hline 32 & CSR 2016-IR18-17 & 100.00 & 102.80 & 14.60 & 12.67 & 20.60 & 120.33 & 79.20 & 24.61 & 3.00 & 27.18 \\
\hline 33 & CSR 2016-IR18-18 & 99.67 & 103.83 & 10.77 & 8.60 & 24.58 & 115.00 & 77.35 & 24.37 & 3.00 & 30.28 \\
\hline 34 & CSR 2016-IR18-2 & 116.33 & 94.00 & 11.17 & 8.17 & 24.70 & 121.67 & 78.02 & 21.57 & 4.33 & 23.75 \\
\hline 35 & CSR 2016-IR18-3 & 107.33 & 85.03 & 11.93 & 9.93 & 20.91 & 128.00 & 85.47 & 24.65 & 3.00 & 26.07 \\
\hline 36 & CSR 2016-IR18-5 & 110.33 & 90.48 & 15.00 & 12.67 & 25.60 & 79.33 & 70.20 & 20.83 & 3.00 & 23.17 \\
\hline 37 & CSR 2016-IR18-7 & 95.33 & 101.23 & 13.07 & 9.37 & 21.04 & 99.00 & 83.14 & 22.43 & 5.00 & 20.38 \\
\hline 38 & CSR 2016-IR18-8 & 96.00 & 102.60 & 11.50 & 9.33 & 24.13 & 124.00 & 75.81 & 22.50 & 2.00 & 30.78 \\
\hline 39 & CSR 2016-IR18-9 & 81.33 & 75.57 & 13.90 & 11.00 & 18.70 & 98.33 & 74.85 & 22.13 & 5.00 & 12.61 \\
\hline 40 & CSR RIL-01-IR165 & 101.67 & 106.40 & 10.77 & 7.93 & 22.23 & 121.00 & 79.11 & 21.88 & 2.00 & 32.93 \\
\hline 41 & CSR-2748-197 & 84.00 & 102.07 & 17.87 & 13.93 & 22.75 & 120.33 & 77.19 & 20.77 & 2.00 & 30.88 \\
\hline 42 & CSR-2748-4441-193 & 77.00 & 125.23 & 9.43 & 7.10 & 23.41 & 118.67 & 82.54 & 23.50 & 1.00 & 35.22 \\
\hline 43 & CSR-2748-4441-195 & 95.00 & 82.93 & 14.60 & 11.97 & 22.46 & 119.67 & 75.47 & 18.73 & 2.00 & 27.26 \\
\hline 44 & CSRC(S)47-7-B-B-1-1 & 87.00 & 137.87 & 11.17 & 7.57 & 20.56 & 102.67 & 77.49 & 19.03 & 3.00 & 30.90 \\
\hline 45 & CSR-C27SM-117 & 92.00 & 96.27 & 12.93 & 10.73 & 18.25 & 105.00 & 72.18 & 24.90 & 2.00 & 33.95 \\
\hline 46 & $\begin{array}{l}\text { IR 83421-6-B-3-1-1 CR } \\
\text { 3364-S-2 }\end{array}$ & 96.33 & 91.20 & 14.77 & 11.20 & 20.69 & 119.00 & 76.51 & 23.53 & 3.00 & 21.30 \\
\hline 47 & IR10206-29-2-1-1 & 105.67 & 105.80 & 10.77 & 7.50 & 19.88 & 120.00 & 79.06 & 18.07 & 7.00 & 16.90 \\
\hline 48 & IR52280-117-1-1-3 & 93.33 & 128.30 & 12.87 & 9.40 & 25.31 & 116.00 & 72.27 & 22.55 & 2.00 & 34.62 \\
\hline 49 & $\begin{array}{l}\text { IR84649-81-4-1-3B- } \\
\text { CR3397-S-B-4 }\end{array}$ & 96.00 & 95.27 & 7.23 & 5.50 & 19.80 & 108.33 & 81.69 & 22.69 & 3.00 & 28.51 \\
\hline 50 & KR 15010 & 78.00 & 74.97 & 10.50 & 8.77 & 24.94 & 129.33 & 79.04 & 17.73 & 7.00 & 17.92 \\
\hline 51 & KR 15016 & 82.00 & 87.17 & 11.80 & 9.23 & 17.48 & 136.00 & 79.81 & 18.77 & 5.00 & 20.18 \\
\hline 52 & KR15006 & 78.67 & 90.83 & 15.77 & 12.77 & 25.92 & 134.00 & 83.16 & 25.23 & 3.00 & 29.33 \\
\hline 53 & KS - 12 & 84.00 & 70.03 & 14.53 & 11.50 & 21.79 & 113.00 & 84.86 & 25.24 & 1.00 & 38.12 \\
\hline
\end{tabular}




\begin{tabular}{|c|c|c|c|c|c|c|c|c|c|c|c|}
\hline 54 & NDRK 11-20 & 86.00 & 94.50 & 16.13 & 12.03 & 21.04 & 123.67 & 76.29 & 23.37 & 2.00 & 29.08 \\
\hline 55 & NDRK 11-21 & 86.00 & 92.77 & 12.17 & 9.60 & 21.44 & 99.00 & 75.46 & 27.23 & 3.00 & 29.36 \\
\hline 56 & NDRK 11-22 & 94.33 & 100.23 & 12.93 & 10.27 & 22.07 & 103.00 & 67.10 & 24.67 & 3.00 & 20.91 \\
\hline 57 & NDRK 11-24 & 84.67 & 91.17 & 10.97 & 8.47 & 23.65 & 124.67 & 76.73 & 25.43 & 2.00 & 31.66 \\
\hline 58 & PAU 3835-12-1-1-1 & 103.33 & 86.94 & 16.00 & 11.67 & 20.74 & 121.33 & 82.45 & 22.00 & 5.00 & 22.03 \\
\hline 59 & PAU 3835-36-6-3-3-4 & 84.67 & 77.53 & 10.43 & 8.43 & 23.80 & 109.67 & 69.57 & 21.70 & 5.00 & 18.40 \\
\hline 60 & PAU 4254-14-1-2-2-2-4-1 & 115.33 & 101.40 & 12.43 & 9.83 & 21.26 & 114.33 & 72.53 & 21.07 & 5.00 & 16.38 \\
\hline 61 & PAU 5563-23-1-1 & 108.00 & 75.53 & 10.27 & 8.33 & 20.46 & 104.33 & 71.37 & 18.50 & 5.00 & 26.08 \\
\hline 62 & PAU 7114-3480-1-1-1-0 & 85.67 & 103.70 & 12.17 & 10.00 & 22.07 & 140.00 & 80.51 & 20.90 & 2.00 & 28.05 \\
\hline 63 & RAU 1397-14 & 104.67 & 85.23 & 15.33 & 12.60 & 19.60 & 111.00 & 79.52 & 20.40 & 2.00 & 32.86 \\
\hline 64 & RP 5440-302-100-7-6-3-2 & 110.00 & 66.40 & 13.53 & 9.27 & 19.98 & 113.00 & 79.09 & 18.07 & 5.00 & 16.25 \\
\hline 65 & RP 5687-420-111-5-4-2-1 & 115.00 & 100.87 & 14.13 & 8.40 & 22.63 & 140.33 & 81.22 & 19.70 & 7.00 & 18.23 \\
\hline 66 & RP 5694-36-9-5-1-1 & 109.00 & 107.07 & 16.80 & 13.53 & 23.13 & 113.00 & 76.76 & 20.85 & 5.00 & 17.80 \\
\hline 67 & RP-320-4-3-2-1 & 115.33 & 67.27 & 11.53 & 7.67 & 24.29 & 156.33 & 75.90 & 18.80 & 7.00 & 10.85 \\
\hline 68 & RP-5683-101-85-30-2-3-1 & 96.00 & 71.53 & 10.27 & 8.70 & 18.21 & 113.00 & 78.15 & 23.00 & 3.00 & 19.86 \\
\hline 69 & Sambha Sub1 & 120.00 & 87.97 & 15.43 & 12.83 & 18.71 & 128.00 & 77.64 & 22.58 & 7.00 & 19.59 \\
\hline 70 & TR 09027 & 87.00 & 63.87 & 13.33 & 11.10 & 19.60 & 87.33 & 72.42 & 18.63 & 5.33 & 13.05 \\
\hline 71 & TR 09030 & 95.67 & 87.53 & 11.60 & 9.20 & 21.21 & 122.33 & 83.21 & 21.65 & 2.00 & 35.03 \\
\hline 72 & CSR10 @ & 88.67 & 77.03 & 16.93 & 14.73 & 19.29 & 117.33 & 81.76 & 22.03 & 1.78 & 30.43 \\
\hline 73 & CST7-1 (C) & 106.33 & 94.30 & 15.40 & 12.17 & 22.71 & 131.00 & 79.60 & 21.30 & 4.78 & 21.05 \\
\hline 74 & CSR36 ㄷ & 106.67 & 101.07 & 14.30 & 12.00 & 23.14 & 136.00 & 83.61 & 24.50 & 1.56 & 31.59 \\
\hline 75 & CSR27 (C) & 95.67 & 95.60 & 10.23 & 8.17 & 21.88 & 125.67 & 83.51 & 24.33 & 2.00 & 30.85 \\
\hline 76 & Sambha Sub1 @ & 120.67 & 77.73 & 14.13 & 11.70 & 20.03 & 122.67 & 79.94 & 21.60 & 4.78 & 25.83 \\
\hline 77 & UsarDhan 3 ○ & 115.00 & 95.43 & 13.53 & 11.50 & 24.13 & 131.67 & 79.86 & 23.50 & 2.00 & 29.89 \\
\hline \multirow[t]{5}{*}{78} & PUSA44 (C) & 93.67 & 79.23 & 10.27 & 8.17 & 20.10 & 115.00 & 76.51 & 19.60 & 7.00 & 18.21 \\
\hline & Mean & 99.20 & 94.12 & 12.56 & 9.74 & 21.63 & 117.44 & 77.04 & 21.77 & 3.75 & 24.73 \\
\hline & C.V. & 2.74 & 2.63 & 7.08 & 9.87 & 3.10 & 18.48 & 5.90 & 2.58 & 6.26 & 20.06 \\
\hline & C.D. $5 \%$ & 4.38 & 3.99 & 1.43 & 1.55 & 1.08 & 35.00 & 7.32 & 0.90 & 0.38 & 8.38 \\
\hline & C.D. $1 \%$ & 5.79 & 5.27 & 1.89 & 2.04 & 1.43 & 46.22 & 9.68 & 1.19 & 0.50 & 10.59 \\
\hline
\end{tabular}

Table.2 Analysis of variance Augmented R.B.D. (Summary) for 78 genotypes for 10 characters in Oryza sativa in alkaline soil

\begin{tabular}{|c|c|c|c|c|c|c|c|c|c|c|c|}
\hline $\begin{array}{l}\text { Source of } \\
\text { variance }\end{array}$ & DF & $\begin{array}{c}\text { Days to } \\
50 \% \\
\text { flowering }\end{array}$ & $\begin{array}{c}\text { Plant } \\
\text { height } \\
(\mathbf{c m})\end{array}$ & Tillers/Plant & $\begin{array}{c}\text { Productive } \\
\text { Tillers/plant }\end{array}$ & $\begin{array}{c}\text { Panicle } \\
\text { Length } \\
\text { (cm) }\end{array}$ & $\begin{array}{c}\text { Filled } \\
\text { grains/panicle }\end{array}$ & $\begin{array}{c}\text { Spikelet } \\
\text { Fertility } \\
(\%)\end{array}$ & $\begin{array}{c}\text { Test } \\
\text { Weight }\end{array}$ & $\begin{array}{c}\text { Stress score } \\
\text { at } \\
\text { reproductive } \\
\text { stage }\end{array}$ & $\begin{array}{c}\text { Grain } \\
\text { Yield } \\
\text { g/plant }\end{array}$ \\
\hline $\begin{array}{l}\text { Block (ignoring } \\
\text { Treatments) }\end{array}$ & 2.00 & 11.50 & $371.15^{* *}$ & $28.50^{* *}$ & 3.58 & 16.48 & 428.20 & $45.30^{* *}$ & $2.65 * *$ & $4.66^{* * *}$ & 48.20 ** \\
\hline $\begin{array}{l}\text { Treatment } \\
\text { (eliminating } \\
\text { Blocks) }\end{array}$ & 78.00 & $132.50 * * *$ & $172.50^{* *}$ & 6.51 & 5.62 & 5.08 & 618.40 & $59.38 * * *$ & $7.85^{* * *}$ & 1.93 & $27.12 * *$ \\
\hline Checks & 7.00 & $253.00 * * *$ & $380.25 * *$ & 19.50 ** & $16.89 * *$ & 6.58 & 861.25 & $50.89 * *$ & $9.27 * * *$ & 2.10 & $30.08 * *$ \\
\hline $\begin{array}{l}\text { Checks+Var } \\
\text { vs.Var. }\end{array}$ & 72.00 & $123.50 * * *$ & $151.40^{* *}$ & 6.30 & 3.77 & 5.83 & 599.25 & $60.06 * * *$ & $7.74 * * *$ & 1.92 & $26.88 * *$ \\
\hline ERROR & 13.00 & 16.50 & 45.20 & 5.78 & 4.08 & 6.52 & 215.57 & 7.38 & 0.23 & 0.86 & 6.20 \\
\hline $\begin{array}{l}\text { Block (eliminating } \\
\text { Check+Var.) }\end{array}$ & 2.00 & 47.25 & 105.20 & 1.67 & 1.22 & 5.63 & 58.91 & 14.57 & 0.06 & 0.19 & 5.63 \\
\hline $\begin{array}{l}\text { Entries (ignoring } \\
\text { Blocks) }\end{array}$ & 78.00 & 131.24 & $172.00^{* *}$ & 7.68 & 4.82 & 5.32 & 730.26 & $60.17 * * *$ & $7.92 * * *$ & $2.05^{* *}$ & $28.23 * *$ \\
\hline Checks & 7.00 & 253.20 *** & $380.25 * *$ & $20.15^{* *}$ & $16.89 * *$ & 6.51 & 961.52 & $50.89 * *$ & $9.27 * * *$ & 2.10 & $30.08 * *$ \\
\hline Varieties & 71.00 & $123.25^{* * * *}$ & $148.25^{* *}$ & 6.67 & 4.50 & 5.28 & 718.40 & $60.71 * * *$ & $7.65^{* * *}$ & $2.06^{* *}$ & $27.23 * *$ \\
\hline $\begin{array}{l}\text { Checks vs. } \\
\text { Varieties }\end{array}$ & 1.00 & 48.30 & $830.40 * *$ & $35.80 * *$ & $27.80^{* *}$ & 1.78 & 220.34 & $76.03^{* *}$ & $20.06^{* * *}$ & 0.92 & $91.45^{* *}$ \\
\hline ERROR & 13.00 & 16.20 & 45.30 & 6.30 & 4.08 & 5.48 & 215.57 & 7.38 & 0.23 & 0.86 & 6.20 \\
\hline $\mathbf{C i}-\mathbf{C} \mathbf{j}$ & 1.00 & 6.70 & 10.30 & 4.10 & 3.58 & 3.52 & 26.12 & 4.83 & 0.85 & 1.65 & 4.43 \\
\hline BiVi - BiVj & 1.00 & 11.33 & 19.50 & 7.08 & 5.63 & 6.58 & 45.24 & 8.37 & 1.47 & 2.85 & 7.67 \\
\hline $\mathbf{B i V i}-\mathbf{B j V j}$ & 1.00 & 12.40 & 18.25 & 8.75 & 6.71 & 7.38 & 48.36 & 8.95 & 1.57 & 3.05 & 8.20 \\
\hline $\mathbf{C i}$ - VI & 1.00 & 10.33 & 17.50 & 5.68 & 5.08 & $7 . .78$ & 39.49 & 7.31 & 1.28 & 2.49 & 6.70 \\
\hline
\end{tabular}


Table.3 Genotypic and Phenotypic correlation among 10 different characters in indica rice genotype

\begin{tabular}{|c|c|c|c|c|c|c|c|c|c|c|c|}
\hline Character & & $\begin{array}{c}\text { Days to } \\
50 \% \\
\text { flowering }\end{array}$ & $\begin{array}{c}\text { Plant } \\
\text { height } \\
\text { (cm) }\end{array}$ & Tillers/Plant & $\begin{array}{l}\text { Productive } \\
\text { Tillers/plant }\end{array}$ & $\begin{array}{l}\text { Panicle } \\
\text { Length } \\
(\mathrm{cm})\end{array}$ & $\begin{array}{c}\text { Filled } \\
\text { grains/panicle }\end{array}$ & $\begin{array}{c}\text { Spikelet } \\
\text { Fertility } \\
\quad(\%)\end{array}$ & $\begin{array}{c}\text { Test } \\
\text { Weight }\end{array}$ & $\begin{array}{c}\text { Stress score } \\
\text { at } \\
\text { reproductive } \\
\text { stage }\end{array}$ & $\begin{array}{l}\text { Grain } \\
\text { Yield } \\
\text { g/plant }\end{array}$ \\
\hline $\begin{array}{c}\text { Days TO } \\
\mathbf{5 0 \%} \\
\text { Flowering }\end{array}$ & $\begin{array}{l}\mathrm{G} \\
\mathrm{P}\end{array}$ & $\begin{array}{l}1.0000 \\
1.0000\end{array}$ & $\begin{array}{l}-0.0754 \\
-0.0642\end{array}$ & $\begin{array}{l}0.0174 \\
0.0255\end{array}$ & $\begin{array}{l}-0.0387 \\
-0.0322\end{array}$ & $\begin{array}{l}-0.0013 \\
0.0028\end{array}$ & $\begin{array}{l}-0.1842 \\
-0.0642\end{array}$ & $\begin{array}{l}-0.1765 \\
-0.1150\end{array}$ & $\begin{array}{c}-0.1426 \\
- \\
0.1398 * *\end{array}$ & $\begin{array}{c}0.5135 \\
0.5000 * * *\end{array}$ & $\begin{array}{c}-0.5147 \\
-0.4072 \\
* * *\end{array}$ \\
\hline $\begin{array}{l}\text { Plant Height } \\
\text { (cm) }\end{array}$ & $\begin{array}{l}\mathrm{G} \\
\mathrm{P}\end{array}$ & & $\begin{array}{l}1.0000 \\
1.0000\end{array}$ & $\begin{array}{l}0.0418 \\
0.0325\end{array}$ & $\begin{array}{l}0.0233 \\
0.0165\end{array}$ & $\begin{array}{c}0.1603 \\
0.1502 * *\end{array}$ & $\begin{array}{l}0.1222 \\
0.0335\end{array}$ & $\begin{array}{l}0.0889 \\
0.0279\end{array}$ & $\begin{array}{c}0.1473 \\
0.1367 * *\end{array}$ & $\begin{array}{c}-0.3369 \\
-0.3305 * * *\end{array}$ & $\begin{array}{c}0.4372 \\
0.3447 * * *\end{array}$ \\
\hline $\begin{array}{r}\text { Tiller } \\
\text { plant }^{-1}\end{array}$ & $\begin{array}{l}\mathrm{G} \\
\mathrm{P}\end{array}$ & & & $\begin{array}{l}1.0000 \\
1.0000\end{array}$ & $\begin{array}{l}0.9227 \\
0.8874\end{array}$ & $\begin{array}{l}0.0722 \\
0.0693\end{array}$ & $\begin{array}{c}0.1143 \\
0.1316 * *\end{array}$ & $\begin{array}{c}0.2043 \\
0.1996 \\
* *\end{array}$ & $\begin{array}{l}0.0397 \\
0.0514\end{array}$ & $\begin{array}{l}-0.1103 \\
-0.1060\end{array}$ & $\begin{array}{l}0.0771 \\
0.1118\end{array}$ \\
\hline $\begin{array}{l}\text { Productive } \\
\text { Tillers plant } \\
-1\end{array}$ & $\begin{array}{l}\mathrm{G} \\
\mathrm{P}\end{array}$ & & & & $\begin{array}{l}1.0000 \\
1.0000\end{array}$ & $\begin{array}{l}0.0024 \\
0.0129\end{array}$ & $\begin{array}{c}0.1353 \\
0.1716 * *\end{array}$ & $\begin{array}{c}0.2600 \\
0.2591 \\
* * *\end{array}$ & $\begin{array}{l}0.1264 \\
0.1257\end{array}$ & $\begin{array}{c}-0.2053 \\
-0.1860 * *\end{array}$ & $\begin{array}{l}0.1751 \\
0.1920\end{array}$ \\
\hline $\begin{array}{c}\text { Panicle } \\
\text { Length }(\mathrm{cm})\end{array}$ & $\begin{array}{l}\mathrm{G} \\
\mathrm{P}\end{array}$ & & & & & $\begin{array}{l}1.0000 \\
1.0000\end{array}$ & $\begin{array}{l}0.0545 \\
0.0543\end{array}$ & $\begin{array}{l}-0.0680 \\
-0.0206\end{array}$ & $\begin{array}{l}0.0878 \\
0.0715\end{array}$ & $\begin{array}{l}-0.1105 \\
-0.1044\end{array}$ & $\begin{array}{l}0.1324 \\
0.1140\end{array}$ \\
\hline $\begin{array}{l}\text { Filled grains } \\
\text { panicle }^{-1}\end{array}$ & $\begin{array}{l}\mathrm{G} \\
\mathrm{P}\end{array}$ & & & & & & $\begin{array}{l}1.0000 \\
1.0000\end{array}$ & $\begin{array}{c}0.7002 \\
0.6124 \\
* * *\end{array}$ & $\begin{array}{l}-0.1850 \\
-0.0617\end{array}$ & $\begin{array}{l}-0.2733 \\
-0.1118\end{array}$ & $\begin{array}{c}0.2182 \\
0.4234 \\
* * *\end{array}$ \\
\hline $\begin{array}{c}\text { Spikelet } \\
\text { Fertility \% }\end{array}$ & $\begin{array}{l}\mathrm{G} \\
\mathrm{P}\end{array}$ & & & & & & & $\begin{array}{l}1.0000 \\
1.0000\end{array}$ & $\begin{array}{l}0.0975 \\
0.0853\end{array}$ & $\begin{array}{c}-0.2705 \\
-0.1710 * *\end{array}$ & $\begin{array}{c}0.3190 \\
0.3920 \\
* * *\end{array}$ \\
\hline Test Weight & $\begin{array}{l}\mathrm{G} \\
\mathrm{P}\end{array}$ & & & & & & & & $\begin{array}{l}1.0000 \\
1.0000\end{array}$ & $\begin{array}{c}-0.4352 \\
-0.4197 * * *\end{array}$ & $\begin{array}{c}0.4057 \\
0.3331 \\
* * *\end{array}$ \\
\hline $\begin{array}{l}\text { Stress Score } \\
\text { at } \\
\text { reproductive } \\
\text { stage }\end{array}$ & $\begin{array}{l}\mathrm{G} \\
\mathrm{P}\end{array}$ & & & & & & & & & $\begin{array}{l}1.0000 \\
1.0000\end{array}$ & $\begin{array}{c}-0.9630 \\
-0.7935 \\
* * *\end{array}$ \\
\hline $\begin{array}{c}\text { Grain Yield } \\
\text { Kg/ha }\end{array}$ & $\begin{array}{l}\mathrm{G} \\
\mathrm{P}\end{array}$ & & & & & & & & & & $\begin{array}{l}1.0000 \\
1.0000\end{array}$ \\
\hline
\end{tabular}


Table.4 Total variance explained by different principal component

\begin{tabular}{|c|c|c|c|}
\hline Principal components & Eigen value & Percent variability & Cumulative variability \\
\hline $\mathbf{1}$ & 3.349 & 33.485 & 33.485 \\
\hline $\mathbf{2}$ & 1.375 & 13.752 & 47.237 \\
\hline $\mathbf{3}$ & 1.175 & 11.750 & 58.987 \\
\hline $\mathbf{4}$ & 1.034 & 10.339 & 69.326 \\
\hline $\mathbf{5}$ & 1.071 & 10.713 & 80.038 \\
\hline $\mathbf{6}$ & 0.733 & 7.332 & 87.370 \\
\hline $\mathbf{7}$ & 0.427 & 5.392 & 92.762 \\
\hline $\mathbf{8}$ & 0.103 & 3.310 & 96.072 \\
\hline $\mathbf{9}$ & 0.051 & 2.315 & 98.387 \\
\hline $\mathbf{1 0}$ & 0.003 & 1.613 & 100.000 \\
\hline
\end{tabular}

Table.5 Principal factor analysis; factor loading of different characters (unrotated)

\begin{tabular}{|c|c|c|c|c|c|}
\hline & PCI & PCII & PCIII & PCIV & $\mathbf{P C V}$ \\
\hline Eigene Value (Root) & 3.349 & 1.375 & 1.175 & 1.034 & 1.071 \\
\hline \% Var. Exp. & 33.485 & 13.752 & 11.750 & 10.339 & 10.713 \\
\hline Cum. Var. Exp. & 33.485 & 47.237 & $\mathbf{5 8 . 9 8 7}$ & 69.326 & 80.038 \\
\hline Days to $50 \%$ Flowering & 0.266 & 0.125 & 0.531 & 0.320 & 0.029 \\
\hline Plant Height (cm) & -0.491 & 0.142 & 0.116 & 0.191 & 0.172 \\
\hline Tillers Plant $^{-1}$ & -0.211 & -0.148 & -0.479 & 0.625 & 0.341 \\
\hline Productive Tillers Plant $^{-1}$ & 0.229 & -0.375 & 0.359 & 0.302 & 0.219 \\
\hline Panicle Length (cm) & -0.162 & -0.288 & -0.185 & -0.169 & -0.247 \\
\hline Filled Grains Panicle $^{-1}$ & -0.410 & 0.393 & 0.155 & -0.124 & -0.218 \\
\hline Spikelet Fertility (\%) & -0.432 & 0.230 & 0.328 & 0.237 & 0.035 \\
\hline 1000 Seed Weight (g) & -0.209 & -0.537 & 0.368 & 0.043 & -0.363 \\
\hline Stress score at reproductive stage & 0.339 & 0.409 & 0.086 & -0.042 & 0.211 \\
\hline Grain Yield (gm/plant) & -0.227 & -0.237 & 0.196 & -0.525 & 0.721 \\
\hline
\end{tabular}

Table.6 Principal factor analysis; factor loadings (VARIMAX Rotation) of different

\begin{tabular}{|l|c|c|c|c|c|}
\hline & 1 Factor & 2 Factor & 3 Factor & 4 Factor & 5 Factor \\
\hline Root & 0.999 & 1.003 & 0.986 & 0.995 & 1.017 \\
\hline$\square$ (\%) Explained & 9.992 & 10.031 & 9.860 & 9.952 & 10.165 \\
\hline$\square \sim^{\mathbf{2} C u m \text { Explained }}$ & 19.984 & 40.047 & 59.767 & 79.671 & 100.000 \\
\hline Days to 50\% Flowering & -0.107 & -0.181 & 0.775 & 0.019 & 0.014 \\
\hline Plant Height (cm) & -0.547 & -0.030 & -0.015 & 0.106 & 0.113 \\
\hline Tillers/ Plant & -0.093 & -0.046 & -0.164 & 0.795 & 0.156 \\
\hline Productive Tillers / Plant & 0.156 & -0.591 & 0.094 & 0.071 & -0.374 \\
\hline Panicle Length (cm) & 0.054 & -0.006 & 0.071 & 0.014 & 0.810 \\
\hline Filled Grains/ Panicle & -0.507 & 0.197 & -0.154 & -0.219 & -0.202 \\
\hline Spikelet Fertility (\%) & -0.614 & -0.085 & 0.136 & 0.027 & -0.046 \\
\hline 1000 Seed Weight (g) & -0.100 & -0.641 & -0.022 & -0.117 & 0.195 \\
\hline Stress score at reproductive stage & 0.081 & 0.372 & 0.521 & -0.080 & 0.045 \\
\hline Grain Yield (gm/plant) & -0.047 & -0.154 & -0.173 & -0.527 & 0.317 \\
\hline
\end{tabular}

*factor loading are significant criteria being $\approx 0.60$ 
Table.7 Principal factor score of 71 genotype and 7 check in three principal components

\begin{tabular}{|c|c|c|c|c|}
\hline & Genotype & PCA I & PCA II & PCA III \\
\hline & & X Vector & Y Vector & Z Vector \\
\hline 1. & CARI Dhan 10 & -17.139 & -8.065 & 19.790 \\
\hline 2. & CARI Dhan 11 & -30.640 & -8.841 & 19.886 \\
\hline 3. & CARI Dhan 6 & -20.240 & -10.665 & 24.249 \\
\hline 4. & CR 2851-S-1-6-B-B-4 & -8.365 & -6.026 & 24.826 \\
\hline 5. & CR 2851-S-B-1-2B-1 & -17.354 & -10.312 & 21.063 \\
\hline 6. & CR 2851-S-B-1-B-B-1 & -12.864 & -6.095 & 26.051 \\
\hline 7. & CR 3437-1*-S-200-83-1 & -14.040 & -5.669 & 21.102 \\
\hline 8. & CR 3878-245-2-4-1 & -18.643 & -9.484 & 22.069 \\
\hline 9. & CR 3880-10-1-9-2-2-1 & -14.575 & -7.596 & 20.611 \\
\hline 10. & CR 3881-4-1-3-7-2-3 & -15.028 & -3.840 & 23.867 \\
\hline 11. & CR 3883-3-1-5-2-1-2 & -21.641 & -9.142 & 24.438 \\
\hline 12. & CR 3884-244-8-5-11-1-1 & -10.125 & -7.141 & 22.161 \\
\hline 13. & CR 3887-15-1-2-1 & -20.951 & -7.447 & 20.808 \\
\hline 14. & CR 3890-35-1-3-4 & -22.361 & -11.290 & 21.955 \\
\hline 15. & CR 3904-162-1-5-1 & -11.955 & -6.766 & 28.162 \\
\hline 16. & CR3881-M-3-1-5-1-1-1 & -18.121 & -9.639 & 20.096 \\
\hline 17. & CR3882-7-1-6-2-2-1 & -15.051 & -9.823 & 19.757 \\
\hline 18. & CR3884-244-8-5-6-1-1 & -14.164 & -3.853 & 20.320 \\
\hline 19. & CR3903-161-1-3-2 & -14.543 & -5.791 & 27.319 \\
\hline 20. & CSA 2016-IR18-6 & -23.515 & -11.302 & 22.048 \\
\hline 21. & CSAR 1604 & -15.773 & -11.517 & 22.284 \\
\hline 22. & CSAR 1610 & -18.154 & -12.147 & 21.257 \\
\hline 23. & CSAR 1628 & -16.510 & -8.775 & 25.421 \\
\hline 24. & CSAR 1620 & -17.186 & -12.283 & 21.849 \\
\hline 25. & CSR 2016-IR18-1 & -11.156 & -6.663 & 21.811 \\
\hline 26. & CSR 2016-IR18-10 & -24.208 & -10.482 & 22.052 \\
\hline 27. & CSR 2016-IR18-11 & -15.871 & -6.207 & 23.121 \\
\hline 28. & CSR 2016-IR18-12 & -20.410 & -12.879 & 22.205 \\
\hline 29. & CSR 2016-IR18-14 & -8.944 & -5.333 & 23.165 \\
\hline 30. & CSR 2016-IR18-15 & -16.310 & -10.754 & 20.969 \\
\hline 31. & CSR 2016-IR18-16 & -15.406 & -12.120 & 21.251 \\
\hline 32. & CSR 2016-IR18-17 & -18.961 & -11.314 & 23.041 \\
\hline 33. & CSR 2016-IR18-18 & -20.060 & -12.087 & 23.769 \\
\hline 34. & CSR 2016-IR18-2 & -15.350 & -9.338 & 24.384 \\
\hline 35. & CSR 2016-IR18-3 & -16.030 & -11.529 & 24.501 \\
\hline 36. & CSR 2016-IR18-5 & -15.169 & -12.262 & 21.331 \\
\hline 37. & CSR 2016-IR18-7 & -17.963 & -7.300 & 22.226 \\
\hline 38. & CSR 2016-IR18-8 & -20.300 & -11.919 & 21.881 \\
\hline 39. & CSR 2016-IR18-9 & -13.439 & -8.050 & 18.921 \\
\hline 40. & CSR RIL-01-IR165 & -20.628 & -10.347 & 23.098 \\
\hline 41. & CSR-2748-197 & -20.777 & -10.633 & 17.109 \\
\hline 42. & CSR-2748-4441-193 & -27.526 & -11.365 & 21.459 \\
\hline 43. & CSR-2748-4441-195 & -15.783 & -10.408 & 18.462 \\
\hline 44. & CSRC(S)47-7-B-B-1-1 & -25.675 & -5.506 & 21.268 \\
\hline 45. & CSR-C27SM-117 & -19.162 & -12.244 & 22.356 \\
\hline 46. & $\begin{array}{l}\text { IR 83421-6-B-3-1-1 CR } \\
336\end{array}$ & -17.245 & -10.576 & 20.915 \\
\hline
\end{tabular}




\begin{tabular}{|c|c|c|c|c|}
\hline 47. & IR10206-29-2-1-1 & -14.892 & -2.122 & 23.347 \\
\hline 48. & IR52280-117-1-1-3 & -25.176 & -11.146 & 21.487 \\
\hline 49. & $\begin{array}{l}\text { IR84649-81-4-1-3B- } \\
\text { CR3397- }\end{array}$ & -18.442 & -9.311 & 24.472 \\
\hline 50. & KR 15010 & -12.399 & -5.510 & 18.224 \\
\hline 51. & KR 15016 & -15.098 & -4.586 & 19.329 \\
\hline 52. & KR15006 & -20.248 & -13.414 & 18.777 \\
\hline 53. & KS -12 & -17.852 & -15.181 & 19.584 \\
\hline 54. & NDRK 11-20 & -19.779 & -11.510 & 18.808 \\
\hline 55. & NDRK 11-21 & -19.531 & -13.351 & 22.450 \\
\hline 56. & NDRK 11-22 & -18.634 & -11.927 & 21.782 \\
\hline 57. & NDRK 11-24 & -20.250 & -13.654 & 21.334 \\
\hline 58. & PAU 3835-12-1-1-1 & -14.629 & -7.778 & 21.354 \\
\hline 59. & PAU 3835-36-6-3-3-4 & -13.926 & -9.684 & 19.961 \\
\hline 60. & PAU 4254-14-1-2-2-2-4-1 & -14.769 & -7.301 & 24.183 \\
\hline 61. & PAU 5563-23-1-1 & -10.767 & -7.289 & 22.637 \\
\hline 62. & PAU 7114-3480-1-1-1-0 & -20.941 & -9.744 & 19.957 \\
\hline 63. & RAU 1397-14 & -15.732 & -10.515 & 20.992 \\
\hline 64. & RP 5440-302-100-7-6-3-2 & -9.452 & -6.061 & 20.683 \\
\hline 65. & RP 5687-420-111-5-4-2-1 & -14.534 & -3.569 & 22.898 \\
\hline 66. & RP 5694-36-9-5-1-1 & -16.583 & -7.756 & 21.724 \\
\hline 67. & RP-320-4-3-2-1 & -7.954 & -5.471 & 22.031 \\
\hline 68. & RP-5683-101-85-30-2-3-1 & -13.449 & -10.442 & 22.466 \\
\hline 69. & Sambha Sub1 & -10.784 & -6.358 & 25.069 \\
\hline 70. & TR 09027 & -9.799 & -7.156 & 18.227 \\
\hline 71. & TR 09030 & -17.977 & -10.924 & 21.723 \\
\hline 72. & CSR10 @ & -16.021 & -11.992 & 18.718 \\
\hline 73. & CST7-1 (C) & -15.322 & -8.264 & 21.706 \\
\hline 74. & CSR36 @ & -20.008 & -13.169 & 23.312 \\
\hline 75. & CSR27 (C) & -19.879 & -11.980 & 23.408 \\
\hline 76. & Sambha Sub1 @ & -11.074 & -8.849 & 24.388 \\
\hline 77. & Usar Dhan 3 ○ & -17.581 & -13.054 & 23.998 \\
\hline 78. & PUSA44 ( & -11.487 & -4.921 & 21.809 \\
\hline
\end{tabular}

Fig.1 SCREE Plot graphical representation

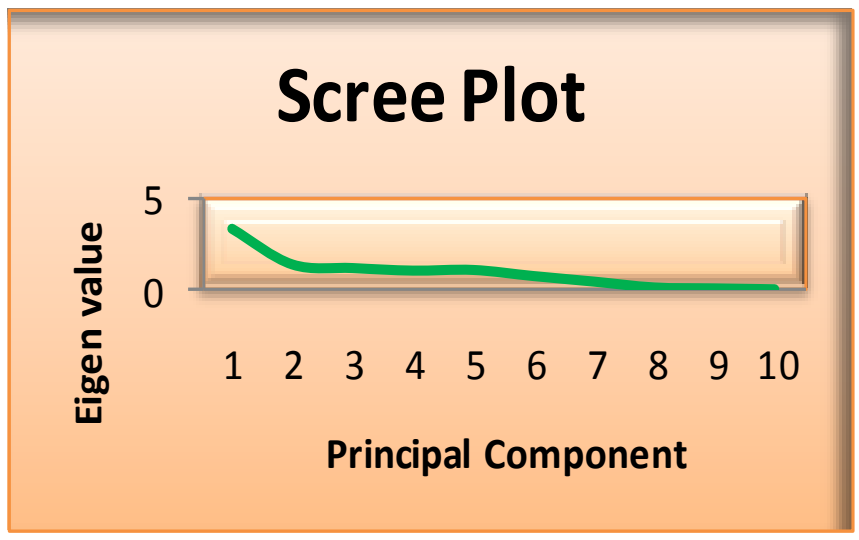


Fig.2 Principal factor 1 and 2 plotting

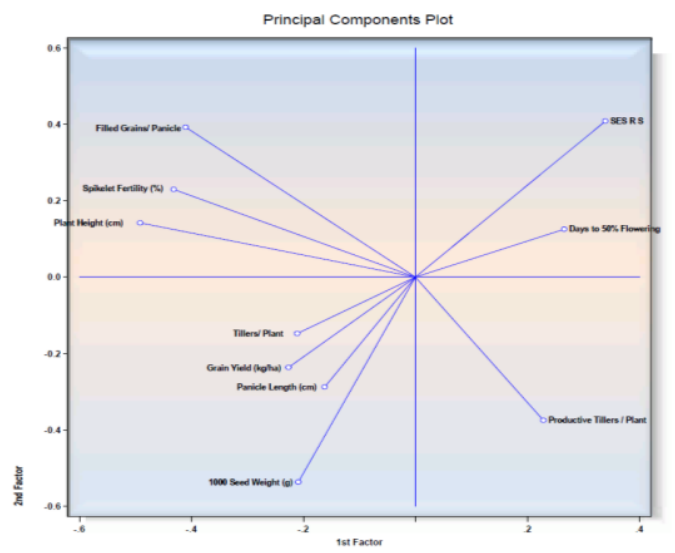

Fig.3 Character association VARIMAX Rotation

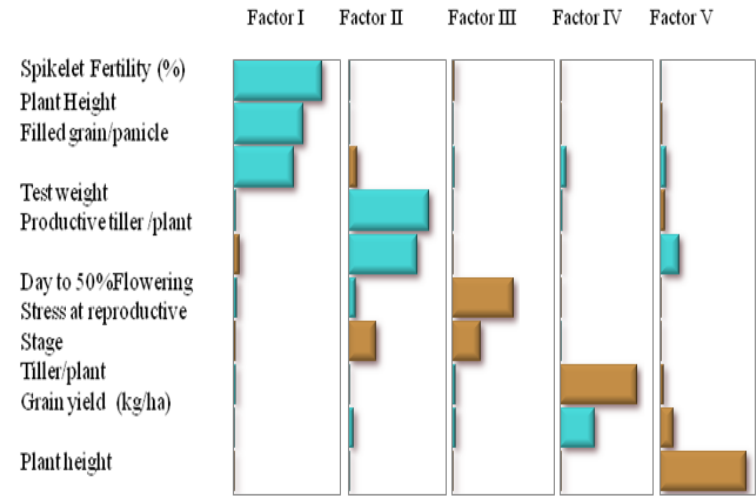

For PC 1 had high positive component loading from Stress score at reproductive stage (0.339), Days to 50\% Flowering (0.266) and Productive Tillers Plant ${ }^{-1}(0.229)$ whereas plant height (-0.491), spikelet Fertility (\%) (-0.432), filled grain panicle ${ }^{-1}(-$ $0.410)$, grain yield $(-0.227), 1000$ seed weight (g) exhibited high negative loading; contributed mostly towards 33.485 percent of total variability. In the second principal component (PC-II) high positive component loading was observed from Stress score at reproductive stage (0.409), Filled Grains Panicle ${ }^{-1}(0.393)$, Spikelet Fertility (\%) (0.230), Plant Height (cm) (0.142) and Days to $50 \%$ Flowering $(0.125)$ followed by high negative loading from thousand grain weight
$(-0.537)$ productive tiller plant ${ }^{-1}(-0.375)$, panicle length $(-0.288)$, grain yield $(-0.237)$ and tiller plant ${ }^{-1}(-0.148)$ are the traits majorly contributing to variability (13.752) created by PC 2.

Total variability (11.750) created by PC 3 were due to the traits Days to $50 \%$ Flowering (0.531), Test Weight (g) (0.368), Productive Tillers Plant-1 (0.359), Spikelet Fertility (\%) (0.328), Plant Height (cm) (0.116), Filled Grains Panicle ${ }^{-1}(0.155)$ Stress score at reproductive stage $(0.086)$ for positive loadings and tiller plant ${ }^{-1}(-0.479)$ and panicle length (-0.185) showed the high negative values. From the three PCs it was clear that among all the ten variables, Stress score at 
reproductive stage, Days to $50 \%$ Flowering and Productive Tillers Plant ${ }^{-1}$ had high value which is agreement with Chahal and Gosal, 2002 who showed that characters with largest absolute value closer to unity within the first principal component influence the clustering more than those with lower absolute value closer to zero. From this study, it was concluded that a good hybridization breeding program can be initiated by the selection of genotypes from the $\mathrm{PC} 1$ as it contributed maximum toward diversity with maximum eigene value (Table 5 and Figure 2).

The failure of principal factor analysis without rotations to give the precise idea of character association with respective principal factor dedicate next alternative factor analysis i.e. with varimax rotation factor loading of different traits with varimax rotation are presented in Table 6. Result from Table 6 and Figure 3 was clearly reflected high loading of spikelet fertility (-0.614), filled grain panical ${ }^{-1}$ $(-0.507)$, plant height $(-0.547)$ on the first factor (PF1). The second factor (PF2) showed high loading of test weight and productive tiller plant ${ }^{-1}$. Days to $50 \%$ flowering $(0.775)$ and stress score at reproductive stage $(0.521)$. Among these principal factor, factor 1 (PF1) can be interpreted as spikelet fertility factor while factor 2 and 3 can be designated as test weight factor and days to $50 \%$ flowering, respectively.

Principal factor score for all the genotype were estimated in all three factors. These score can be utilized to purpose precise selection indices whose intensity can be decided by variability explained by each of principal factor. High principal score for a particular genotype in a particular factor denoted high values for the variable that particular principal factor is representing in that particular genotype.

Critical Perusal of Table 4 reflected that genotype CARI Dhan 11, CSRC(S) 47-7-B-
B-1-1, IR52280-117-1-1-3, CSR 2016-IR1810, and CSA 2016-IR18-6 had high Principal Factor score in factor 1 for spikelet fertility (\%). Similarly, NDRK 11-24, KR15006, NDRK 11-21, CSR36 (C), Usar Dhan 3 (C) had high variation for factor 2 which representing the productive tillers plant $^{-1}$, 1000 seed weight $(\mathrm{g})$. The factor 3 which represent variation for the days to $50 \%$ flowering included genotype CR 3904-162-15-1, CR3903-161-1-3-2, CR 2851-S-B-1-BB-1, CSAR 1628, CSR 2016-IR18-3 (Table 7).

In conclusion the central idea of principal component analysis is to reduce the dimensionality of a data set in which there are a large number of interrelated variables, while retaining as much as possible of the variation present in the data set. The results of the PCA revealed that the $80.038(\%)$ of the total variability was explained by the first five principal components. CARI Dhan 11, CSRC(S) 47-7-B-B-1-1, IR52280-117-1-1-3, CSR 2016-IR18-10, CSA 2016-IR18-6 6 had high PF score in factor 1 indicating that they had high variation for spikelet fertility (\%). Similarly, PF score NDRK 11-24, KR15006, NDRK 11-21, CSR36 @, Usar Dhan 3 @ were high variation in factor 2 which representing the productive tillers plant-1, 1000 seed weight.

\section{References}

Akhilesh Kumar Yadav, R.P. Vyas, and V.K. Yadav.2016. Study on character association and path coefficient in rice, Progressive Research - An International Journal.11 (4): 498-501.

Anjan Kumar Sinha and P. K. Mishra. 2015. Identification of Important AgroMorphic Characters of Traditional Rice (Oryza sativa L.) of West Bengal for Crop Improvement through Principal Component analysis the ECOSCAN, 9(1\&2): 541-546. 
Annu, B.V.S. Sisodia and V. N. Rai. 2016. An application of principal component analysis for pre- harvest forecast model for rice crop based on biometrical characters Journal of Applied and Natural Science, 8 (3): 1164- 1167.

Ariyo ON. 1987. Mulitvariate analysis and choice of parents in Okra (A. esculentus). Annals of Applied Biology, 116: 335-341.

Ashim Chakravorty, Ghosh, P.D. and Sahu, P.K. 2013. Multivariate analysis of phenotypic diversity of landraces of rice of West Bengal. American Journal of Experimental Agriculture, 3(1): 110-123.

Atul Kumar Pachauri, AK Sarawgi, S Bhandarkar and GC Ojha. 2017. Agromorphological characterization and morphological based genetic diversity analysis of Rice (Oryza sativa L.) germplasm. Journal of Pharmacognosy and Phytochemistry, 6(6): 75-80.

Babar, M., Khan, A.A., Arif, A; Zafar, Y. and Arif, M.2009.Path analysis of some leaf and panicle traits affecting grain yield in double haploid lines of rice (Oryza sativa L), J. Agri. Res.2009, 45 (4): 245-252.

Chahal,G.S. and Gosal, SS.2002.Principal and procedure of plant breeding, biotechnology and conventional approaches. Narosa publication house. Inc., New Delhi India.

Escobar-Hernandez, A., Troyo-dieguez, E., Garcia-hern and ezcontreras, J.L., Murillo-amador, B. and Lopezaguilar, R.2005. Principal component analysis to determine forage potential of salt grass Distichlis spicata L. (Grrene) in coastal ecosystems of Baja Califoniasur, Mexico. Tech. Реси. Mex. 43: 13-25.

FAO. Land and Plant Nutrition Management
Service; 2008. Retrieved from: http://www.fao.org/ag b/agl /agll/spush/.

Flowers T J, Flowers S A. 2005. Why does salinity pose such a difficult problem for plant breeders? Agric Water Manag, 78: 15-24.

Gana A. S., Shaba S. Z. and Tsado E. K. 2013. Principal component analysis of morphological traits in thirty-nine accessions of rice (Oryza sativa L.) grown in a rainfed lowland ecology of Nigeria. Journal of Plant Breeding and Crop Science, Vol. 5(x), pp. 120-126.

Gomez, K.A. and Gomez, K.K. 1984. Statistical analysis for agricultural research, 2nd Ed. John Eilly \& Sons,New York.

Gour, L., Maurya, S.B., Koutu, G.K., Singh, S.K., Shukla, S.S., \& Mishra, D.K. 2017. Characterization of rice (Oryza sativa L.) genotypes using principal component analysis, including scree plot \& rotated component matrix. International Journal of Chemical Studies, 5 (4): 975-983.

Hamsa Poorna Prakash, O.P. Verma, Amit Kumar Chaudhary and Mohammad Amir. 2018. Correlation and Path Coefficient Analysis in Rice (Oryza sativa L.) for Sodicity Tolerance International Journal of Current Microbiology and Applied Sciences. Volume 7 Number 07.

Jayasudha, S. and Sharma, Deepak. Genetic parameters of variability, correlation and path-coefficient for grain yield and physiological traits in rice (Oryza sativa L.) under shallow lowland situation. Electronic Journal of Plant Breeding,2010 1 (5): 1332-1338.

Kalyan, B., K.V. Radha Krishna1 and L.V. Subba Rao. 2017.Correlation Coefficient Analysis for Yield and its Components in Rice (Oryza sativa L.) Genotypes 
Int.J.Curr.Microbiol.App.Sci 6(7): 2425-2430.

Leonard, K. and Peter, R.J. 2009. Finding Groups in Data: An Introduction to Cluster Analysis. pp.344.

Mahendran, R., P. Veerabadhiran, S. Robin \& M. Raveendran. 2015. Principal Component Analysis of Rice germplasm accessions under high temperature Stress IJASR, Vol. 5, Issue 3, pp. 355-360.

Maji AT, Shaibu AA. 2014.Application of principal component analysis for rice germplasm characterization and evaluation Journal of Plant Breeding and Crop Science. 4(6): 87-93.

Manikya M and Reddy TD. 2011. Studies on genetic divergence in medium duration elite rice genotypes (Oryza sativa L.) J. Res. ANGRAU 39.

Nachimuthu VV, Robin S, Sudhakar D, Raveendran M, Rajeswari S, Manonmani S. 2014. Evaluation of Rice Genetic Diversity and Variability in a Population Panel by Principal Component Analysis Indian Journal of Science and Technology. 7(10): 1555-1562.

Nair NV, Balakrishnan R, Sreenivasan TV. 1998. Variability of quantitative traits in exotic hybrid germplasm of sugarcane. Genetic Resources and Crop Evolution, 45: 459-464.

Nayak, A.R., Chaudhary, D. and Reddy, J.N. 2004. Inter-relationship among qualily characters in scented rice. Indian Journal of Agricultural Research, 37 (2): 126-127.

Ojha, GC, AK Sarawgi, Bhawana Sharma and Mangla Parikh. 2017. Principal component analysis of morphophysiological traits in rice germplasm accessions (Oryza sativa L.) under rainfed condition International Journal of Chemical Studies; 5(5): 1875-1877.
Qamar, Zia Ul; Cheema, A. A., Ashraf, M., Rashid, M. and Tahir, G.R. 2005. Association analysis of some yield influencing traits in aromatic and nonaromatic rice. Pak. J. Bot. 37 (3): 613627.

Ratna, M., S. Begum A. Husna S. R. Dey and M. S. Hossain. 2015. correlation and path coefficients analyses Bangladesh J. Agril. Res. 40(1): 153-161 in basmati rice.

Ravikumar, B.N.V.S.R., NagaLumari, P., Rao, V. R. M. P., Girija, R. P.V., Satyanarayana, N., Chamundeswari, K., Mohan V., Suryanarayana, Y., Bharathalakshmi, M., and Reddy, A.V. 2015. Principal component analysis and character association foryield components in rice (Oryza sativa L.) cultivars suitable for irrigated ecosystem. Current Biotica. 9(1): 25-35.

Ravikumar, B.N.V.S.R., P. Naga Kumari, P. Venkata Ramana Rao, M. Girija Rani, P.V. Satyanarayana, N. Chamundeswari, K. Mohan Vishnuvardhan, Y. Suryanarayana,M. Bharathalakshmi and A. Vishnuvardhan Reddy. 2015. Principal component analysis and character association for yield components in rice (Oryza sativa L.) cultivars suitable for irrigated ecosystem ISSN 0973-4031 Current Biotica. 9(1):2535.

Ravindra Babu, V., Shreya, K., Kuldeep Singh, D., Usharani, G. and Siva Shankar, A. 2012. Correlation and path analysis studies in popular rice hybrids of India. International Journal of Scientific and Research Publications, 2(3): 1-5.

Sonu Kumar, MP Chauhan, Amit Tomar, Ravindra Kumar Kasana and Nimit Kumar. 2018. Correlation and path coefficient analysis in rice (Oryza 
sativa L.). Vol. 7, Issue 6.

Sudhir K Pathak, G Roopa Lavanya, G Suresh Babu and Neha Srivastava. 2018. Evaluation of rice germplasm for genetic diversity on yield characters by principal component analysis, The Pharma Innovation Journal; 7(4): 661-664.

Szabolcs, I. Soils and salinisation. In: M. Pessarakli. (Ed.), 1994. Handbook of Plant and Crop Stress. New York: Marcel Dekker. 3-11 pp.

Vishnu, V.N., Robin S., Sudhakar, D., Raveendran, M., Rajeswari, S. And Manonmani, S. 2014. Evaluation of Rice Genetic Diversity and Variability in a Population Panel by Principal Component Analysis. Indian Journal of Science and Technology, 7(10),
1555-1562.

Yadav, V.K., Singh, Y, Soni, S.K., and Yadav A.K. 2011. Genetic Divergence Analysis in salt tolerance rice (Oryza Sativa L.) genotypes Plant Archives. 11(2): 593-595.

Yadav, V.K., Soni, S.K. and Singh, S.P. 2012. Genetic studies for Yield and component Traits in world mini core rice collections under sodic soils, Progressive Research. 7(2):219-222.

Yadav, V.K., Soni, S.K., Singh, D. and Singh, S.P. 2012. Non-Hierarchical Euclidean Cluster Analysis to Design Desirable Salt Tolerance Rice (Oriza sativa L.) Genotypes under Irrigated Ecology, Progressive Research. 7(2):219-222.

\section{How to cite this article:}

Kashyap, A. and Vijay Kumar Yadav. 2020. Principal Component Analysis and Character Association for Yield Components in Rice (Oryza sativa L.) Genotypes of Salt Tolerance under Alkaline Condition. Int.J.Curr.Microbiol.App.Sci. 9(10): 481-495. doi: https://doi.org/10.20546/ijcmas.2020.910.059 